\title{
Fault Location Method for Two-Terminal Untransposed Overhead Lines in Distribution Networks Measured by $\mu$ MPMU
}

\author{
Tao Wen ${ }^{*}$, Fangxu Zhang ${ }^{1}$ and Chengbin Wang ${ }^{1}$ \\ ${ }^{1}$ School of Electrical Engineering, Shandong University, Jinan, Shandong, 250061, China
}

\begin{abstract}
With the rapid development of distribution networks, two-terminal overhead lines have been used on a large scale for higher power supply reliability, thus the fault location has attracted much attention. Accurate fault location is helpful to shorten the outage time and improve the economy of operation greatly. However, since insufficient standardization of equipment selection and poor management, line parameters are usually inaccurate or even unknown, mature fault location methods based on impedance can't be applied anymore. Also, the asymmetry caused by non-transposition in distribution networks affects the accuracy of fault location. This paper proposes a fault location method for two-terminal untransposed overhead lines without requiring line parameters. Firstly, this paper considers parameter asymmetry, and the mutual impedances between the three phases are set as different values. Secondly, the location equations rely on three-phase networks, then the self-impedance and mutual impedances are regarded as unknowns and solved directly. Finally, this method takes the average value of fundamental frequency components from different data windows, which reduces error and improves accuracy. The simulation results show that the fault location method has high accuracy, and can effectively overcome the influence of unknown line parameters and non-transposition.
\end{abstract}

\section{Introduction}

With the rapid growth of electric power systems, the scale of distribution networks is becoming increasingly larger. The distribution network is the last link that directly faces the user. The fault in distribution networks tremendously destroys the safe operation of the power system. Accurate fault location is of great significance to restore power supply.

The existing fault location methods for two-terminal overhead lines can be divided into three categories: traveling wave method, active fault location method and impedance method. The traveling wave method is a transient-based method, which makes use of the propagation characteristics of traveling waves generated when a fault occurs[1]. The load of distribution networks changes frequently, which may cause interference to the method. Because the traveling wave method uses highfrequency transient information, it requires high sampling frequency and high investment cost. The active fault location method is different from the passive method only depending on the measurement data in the fault state. The main principle is to amplify the fault feature by injecting a signal into the system or temporarily changing the neutral grounding mode after the fault, such as the injected signal tracing method. The disadvantage of this method is not only high investment cost but also a certain threat to personal security. The impedance method is a method based on the steady state, which calculates the fault distance by solving the circuit equations. The impedance method can be divided into the single-terminal impedance method and the twoterminal impedance method. When the fault occurs via a fault resistance, the single-terminal location method cannot achieve accurate distance measurement theoretically. The two-terminal fault location method can avoid the error caused by fault resistance in principle, so it has better development and application prospects[2]. Compared with transmission networks, the poor maintenance conditions and non-transposition in distribution networks make the fault location more difficult. References [3-4] convert fault location into an optimization problem and calculate it through iteration, but it is necessary to provide a relatively accurate initial value for line parameters. If the initial value and the actual result differ greatly, a complete error will appear in the final results. Moreover, these two methods are only applicable to fully transposed transmission lines. References [5-6] can only identify the faulty section in untransposed networks, and make the protection device act correctly, but cannot locate the fault point. References [7-8] study the harm caused by untransposed lines in distribution networks and the solutions for asymmetry. These two methods need high investment, and the actual effect is greatly affected by the operation mode, which has certain limitations. References [9-10] solve the phase-mode transformation matrix and then use the mode component theory to locate the fault. In references [11-12], the theory of modular analysis is used

"Corresponding author's e-mail: jstzwentao@mail.sdu.edu.cn 
for fault location. Most of the references above have certain requirements for line parameters in distribution networks, but line parameters in distribution networks are usually inaccurate or even unknown. Adopting the theoretical line parameters in distribution networks will cause large errors. Reference [13] proposes a fault location algorithm without requiring line parameters in transmission networks, which cannot be directly applied to distribution networks.

The emerging $\mu \mathrm{MPMU}$ (micro-multifunctional phasor measurement unit) provides a new technical

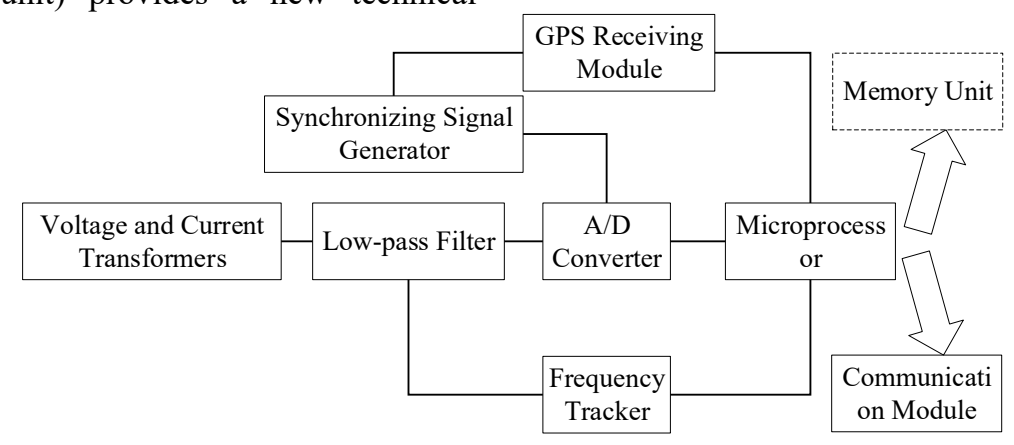

Figure 1. Typical structure of the $\mu \mathrm{MPMU}$

To solve the problems above, this paper proposes a fault location method for untransposed overhead lines based on $\mu$ MPMU. This method has the following advantages: (1) The line impedance parameters are not needed in fault location. Line parameters and the fault distance are taken as unknowns to be calculated together in the equations, so the location results are not affected by inaccurate line parameters and asymmetry. (2) The use of $\mu$ MPMU guarantees the precision of synchronization angle, so this method does not need to consider the problem of unsynchronized sampling results. (3) The fault location method based on two-terminal measurement overcomes the influence of fault resistance, operation mode and system impedance.

In the rest of this paper, Section 2 indicates the establishment and solution of the fault location equations. Section 3 evaluates the performance of the method and a summary is presented in Section 4.

\section{Fault location method}

\subsection{Extraction of fundamental frequency components and filtering algorithm}

In this paper, the voltage and current phasors of fundamental frequency components are used in the location method, so there is a need to calculate the amplitude and phase angle. DFT (Discrete Fourier Transform) algorithm is chosen to extract the fundamental frequency component and the algorithm itself has a filtering function.

In order to further reduce the error and improve the accuracy, based on the characteristics of the fundamental frequency component's amplitude and phase angle, this paper proposes a filtering algorithm of taking the average value of multiple measurements. Firstly, more groups of MPMUs' $^{\prime}$ sampling data can be formed by sliding the method for locating the fault accurately without line parameters. It utilizes the precise timing of GPS to realize synchronous sampling of all points in the network and sends the measurement data with time tags through high-speed communication networks. The typical structure of a $\mu \mathrm{MPMU}$ is shown in figure 1 below. With the improvement of distribution automation level, it is expected that $\mu \mathrm{MPMU}$ will be popularized on a large scale in distribution networks.

sampling data window over one sampling period $\Delta t$. Each fundamental frequency component can be obtained using any one cycle of sampling data based on the DFT algorithm, the amplitude and angle of the fundamental frequency component from the i-th data window are denoted as $A_{i}$ and $\alpha_{i}(i=1,2,3 \ldots n)$. The real amplitude and angle are denoted as $\mathrm{A}$ and $\alpha$. Without error, $\left\{A_{i}\right\}$ is a constant sequence and $A_{1}=A_{2}=\cdots=A_{n}=A$, while $\left\{\alpha_{i}\right\}$ is an arithmetic sequence and the common difference is $h=\omega_{1} \Delta t, \omega_{1}$ means the angular frequency of the fundamental frequency component. It is very clear that $\alpha_{i}=\alpha_{1}+(i-1) h(i=1,2,3 \ldots n)$ and $\alpha=\alpha_{1}$ with no error. Now that random error is inevitable, the calculated value fluctuates around the real value. Therefore, we can reduce error by taking the average value of multiple measurements.

Then the amplitude can be calculated by:

$$
A=\frac{\sum_{i=1}^{n} A_{i}}{n}
$$

As for phase angle, the sum of the arithmetic sequence is shown below:

$$
\sum_{i=1}^{n} \alpha_{i}=n \alpha_{1}+\frac{n(n-1)}{2} \mathrm{~h}
$$

Then,

$$
\alpha=\frac{\sum_{i=1}^{n} \alpha_{i}-\frac{n(n-1)}{2} \mathrm{~h}}{n}
$$

Hence, random error can be restrained very effectively and the accuracy of fault location can be improved based on the filtering algorithm in this section.

\subsection{Establishment of fault location equations}

The diagram of the two-terminal overhead line structure is shown in figure 2 . The neutral point of the system is grounded through a small resistance. Two terminals are 
named terminal $\mathrm{M}$ and $\mathrm{N}$. Considering overhead lines in

neglects the shunt capacitances. distribution networks are usually short, this method

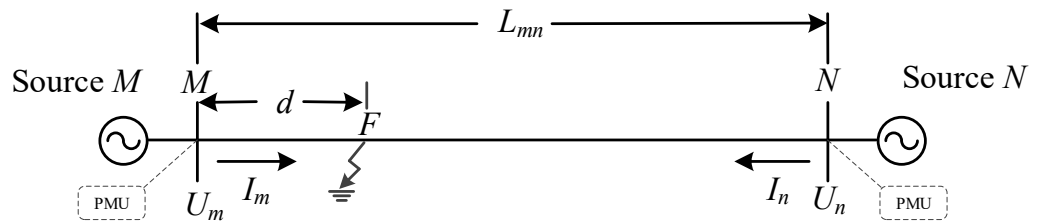

Figure 2. The two-terminal overhead line with a fault

In this paper, the line parameters and fault distance are regarded as unknowns and the impedance matrix is set as

$$
z=\left[\begin{array}{ccc}
z_{s} & z_{a b} & z_{a c} \\
z_{a b} & z_{s} & z_{b c} \\
z_{a c} & z_{b c} & z_{s}
\end{array}\right]
$$

The variables are defined as follows:

$z_{S} \quad$ self-impedance per unit length for all three phases

$z_{a b} \quad$ mutual impedance per unit length between phase A and Phase B

$z_{a c} \quad$ mutual impedance per unit length between phase $\mathrm{A}$ and Phase $\mathrm{C}$

$z_{b c} \quad$ mutual impedance per unit length between phase B and Phase C
The two-terminal fault location method proposed in this paper is based on the length of the whole line provided already and the assumption that the line parameters are evenly distributed along the line. It takes the self-impedance and phase-to-phase mutual impedance as unknowns to be calculated. The location equations are established based on the principle: the voltage at fault point $F$ can be derived from the two terminals along different paths respectively, and the result should be equal in every phase. This principle is suitable for the networks either before or after the fault occurs. Then the fault location equations based on postfault and pre-fault data can be established as equations (5):

$$
\begin{aligned}
& \dot{U}_{m a \_ \text {po }}-\dot{I}_{m a \_ \text {po }} \times z_{s} \times d-\dot{I}_{m b \_ \text {po }} \times z_{a b} \times d-\dot{I}_{m c \_ \text {po }} \times z_{a c} \times d \\
& =\dot{U}_{n a \_ \text {po }}-\dot{I}_{n a \_ \text {po }} \times z_{s} \times\left(L_{m n}-d\right)-\dot{I}_{n b \_ \text {po }} \times z_{a b} \times\left(L_{m n}-d\right)-\dot{I}_{n c \_ \text {po }} \times z_{a c} \times\left(L_{m n}-d\right) \\
& \dot{U}_{m \mathrm{~b} \_ \text {po }}-\dot{I}_{m a \_ \text {po }} \times z_{a b} \times d-\dot{I}_{m b \_ \text {po }} \times z_{s} \times d-\dot{I}_{m c \_ \text {po }} \times z_{b c} \times d \\
& =\dot{U}_{n b \_ \text {po }}-\dot{I}_{n a \_ \text {po }} \times z_{a b} \times\left(L_{m n}-d\right)-\dot{I}_{n b \_ \text {po }} \times z_{s} \times\left(L_{m n}-d\right)-\dot{I}_{n c \_ \text {po }} \times z_{b c} \times\left(L_{m n}-d\right) \\
& \dot{U}_{m c \_p o}-\dot{I}_{m a \_p o} \times z_{a c} \times d-\dot{I}_{m b \_ \text {po }} \times z_{b c} \times d-\dot{I}_{m c \_ \text {po }} \times z_{s} \times d \\
& =\dot{U}_{n c \_ \text {po }}-\dot{I}_{n a \_ \text {po }} \times z_{a c} \times\left(L_{m n}-d\right)-\dot{I}_{n b \_ \text {po }} \times z_{b c} \times\left(L_{m n}-d\right)-\dot{I}_{n c \_ \text {po }} \times z_{s} \times\left(L_{m n}-d\right) \\
& \dot{U}_{\text {ma_pre }}-\dot{I}_{m a \_ \text {pre }} \times z_{s} \times d-\dot{I}_{m b \_ \text {pre }} \times z_{a b} \times d-\dot{I}_{m c \_ \text {pre }} \times z_{a c} \times d \\
& =\dot{U}_{n a \_ \text {pre }}-\dot{I}_{n a \_ \text {pre }} \times z_{s} \times\left(L_{m n}-d\right)-\dot{I}_{n b \_ \text {pre }} \times z_{a b} \times\left(L_{m n}-d\right)-\dot{I}_{n c \_ \text {pre }} \times z_{a c} \times\left(L_{m n}-d\right) \\
& \dot{U}_{m b \_ \text {pre }}-\dot{I}_{m a \_ \text {pre }} \times z_{a b} \times d-\dot{I}_{m b \_ \text {pre }} \times z_{s} \times d-\dot{I}_{m c \_ \text {pre }} \times z_{b c} \times d \\
& =\dot{U}_{n b \_ \text {pre }}-\dot{I}_{n a \_ \text {pre }} \times z_{a b} \times\left(L_{m n}-d\right)-\dot{I}_{n b \_ \text {pre }} \times z_{s} \times\left(L_{m n}-d\right)-\dot{I}_{n c \_ \text {pre }} \times z_{b c} \times\left(L_{m n}-d\right) \\
& \dot{U}_{m c \_ \text {pre }}-\dot{I}_{m a \_ \text {pre }} \times z_{a c} \times d-\dot{I}_{m b \_ \text {pre }} \times z_{b c} \times d-\dot{I}_{m c \_ \text {pre }} \times z_{s} \times d \\
& =\dot{U}_{n c \_ \text {pre }}-\dot{I}_{n a \_ \text {pre }} \times z_{a c} \times\left(L_{m n}-d\right)-\dot{I}_{n b \_ \text {pre }} \times z_{b c} \times\left(L_{m n}-d\right)-\dot{I}_{n c \_ \text {pre }} \times z_{s} \times\left(L_{m n}-d\right)
\end{aligned}
$$

Among them,

$$
\begin{gathered}
\dot{U}_{m a \_p o}, \dot{U}_{m b \_p o}, \dot{U}_{m c \_p o} \\
\dot{I}_{m a \_p o}, \dot{I}_{m b \_p o}, \dot{I}_{m c \_p o} \\
\dot{U}_{n a \_p r e}, \dot{U}_{n b \_p r e}, \dot{U}_{n c \_p r e} \\
\dot{I}_{n a_{-} p r e}, \dot{I}_{n b \_p r e}, \dot{I}_{n c \_p r e} \\
L_{\mathrm{mn}} \\
d
\end{gathered}
$$

The unknown quantities are the fault distance(d), impedance matrix per unit length of line $\left(z_{s}, z_{a b}, z_{a c}, z_{b c}\right)$. The number of unknowns is less than that of equations, at the terminal $\mathrm{M}$ at the terminal $\mathrm{N}$ post-fault power frequency voltage in Phase A, B, C post-fault power frequency current in Phase A, B, C flowing from the terminal $\mathrm{M}$ pre-fault power frequency voltage in Phase A, B, C pre-fault power frequency current in Phase A, B, C flowing from the terminal $\mathrm{N}$ the length of line $\mathrm{MN}$ fault distance, the length of line MF

so the nonlinear equations can be solved using the trustregion algorithm illustrated in Section 2.3 next. 


\subsection{Solving the fault location equations using the trust-region algorithm}

The conventional iterative method is to appropriate the objective function with its Taylor series around the current iteration point and find its descent direction. The basic idea of the trust-region method is to make the neighborhood more proper and the approximation of the
Taylor series more accurate. Compared with the NewtonRaphson method, the trust-region algorithm can guarantee global convergence and higher robustness, so it is preferred to solve complex and nonlinear equations. More information on the trust-region algorithm can be found in Reference[14].

The flowchart of the proposed fault location method is shown in figure 3.

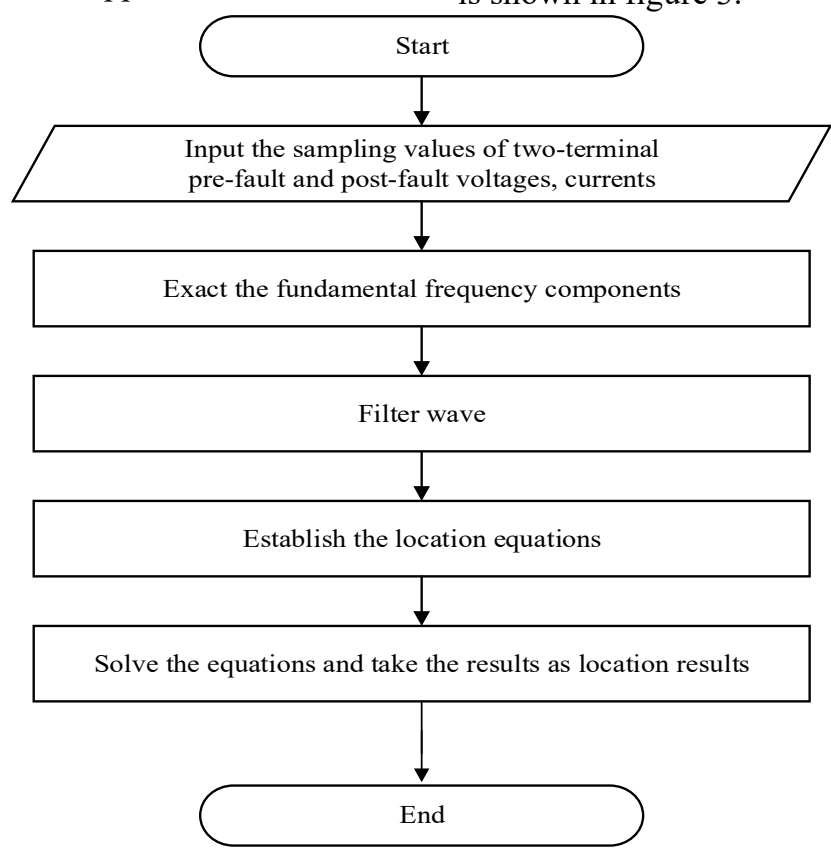

Figure 3. Flow chart of fault location method

\section{Simulation results}

In this paper, a $10 \mathrm{kV}$ two-terminal power supply distribution network model with neutral grounding via low resistance is built in Simulink. The total length of the overhead line is $4 \mathrm{~km}$, which belongs to the short line, so the lumped parameter model is adopted and the shunt capacitance is ignored. The resistance matrix is defined as $R$, the reactance matrix in power frequency is defined as $X$. The line parameters are set as follows:

$\mathrm{R}=\left[\begin{array}{lll}0.0627452 & 0.0415193 & 0.0461325 \\ 0.0415193 & 0.0627452 & 0.0507458 \\ 0.0461325 & 0.0461325 & 0.0461325\end{array}\right] \Omega / \mathrm{km}(6)$

$$
\begin{gathered}
X=\left[\begin{array}{ccc}
0.567624 & 0.242636 & 0.265595 \\
0.242636 & 0.567624 & 0.296555 \\
0.265595 & 0.296555 & 0.567624
\end{array}\right] \Omega / \mathrm{km} \\
Z=R+j X
\end{gathered}
$$

Because of the space limitation, table 1 only presents the results when the fault occurs at the point that is $2 \mathrm{~km}$ from terminal $\mathrm{M}$, more traversal results are presented in figures 4-7. It can be seen that errors are all less than 10 $\mathrm{m}$. The error of fault distance is calculated by (9).

$$
\text { error }_{d}=\left|d_{\text {calculated }}-d_{\text {actual }}\right|
$$

\begin{tabular}{|c|c|c|c|c|c|}
\hline \multirow[b]{2}{*}{$\begin{array}{c}\text { Faulty } \\
\text { Line }\end{array}$} & \multirow{2}{*}{$\begin{array}{c}\text { Fault } \\
\text { Distance } \\
(\mathrm{km})\end{array}$} & \multirow[b]{2}{*}{$\begin{array}{l}\text { Fault } \\
\text { Type }\end{array}$} & \multirow{2}{*}{$\begin{array}{c}\text { Fault } \\
\text { Resistance } \\
(\Omega)\end{array}$} & \multicolumn{2}{|c|}{ Calculated Results } \\
\hline & & & & $\begin{array}{c}\text { Fault Distance } \\
(\mathrm{km})\end{array}$ & $\begin{array}{l}\text { Location Error } \\
\text { (m) }\end{array}$ \\
\hline \multirow{12}{*}{$\mathrm{MN}$} & \multirow{12}{*}{2} & & 1 & 2.0006 & 0.6 \\
\hline & & a-g & 10 & 1.9983 & 1.7 \\
\hline & & & 100 & 2.0095 & 9.5 \\
\hline & & & 1 & 2.0001 & 0.1 \\
\hline & & $a-b$ & 10 & 1.9977 & 2.3 \\
\hline & & & 100 & 2.0068 & 6.8 \\
\hline & & & 1 & 2.0002 & 0.2 \\
\hline & & $a-b-g$ & 10 & 1.9992 & 0.8 \\
\hline & & & 100 & 1.9923 & 7.7 \\
\hline & & & 1 & 1.9998 & 0.2 \\
\hline & & $a-b-c$ & 10 & 2.0031 & 3.1 \\
\hline & & & 100 & 2.0075 & 7.5 \\
\hline
\end{tabular}

Table 1. The representative results of calculated fault distances 


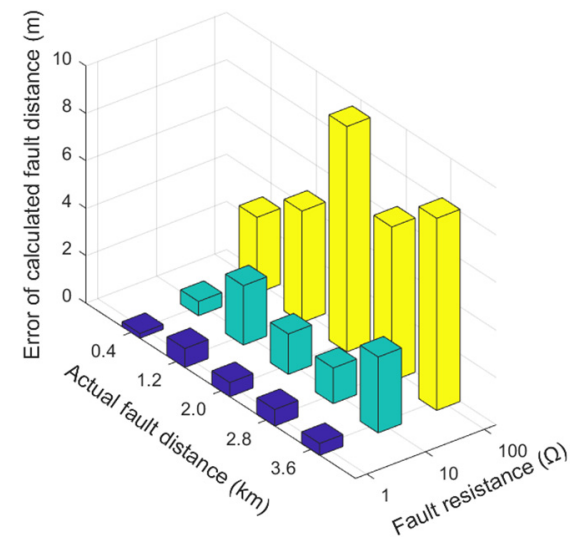

Figure 4. Errors of calculated fault distances for a-g fault

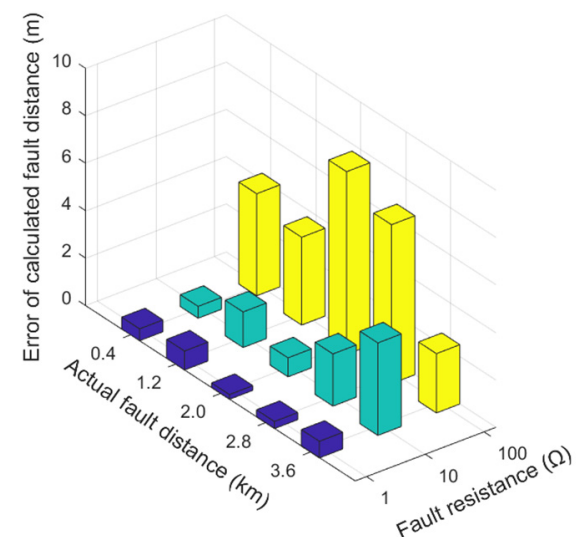

Figure 6. Errors of calculated fault distances for a-b-g fault

\section{Conclusion}

The problem of parameter asymmetry caused by untransposed lines in distribution networks can be solved by the location method proposed in this paper. The method does not require precise line parameters, which is suitable for distribution networks. It is only required that line parameters are uniformly distributed, which overcomes the influence of inaccurate line parameters on traditional location algorithms. The proposed method uses synchronous measurements of the voltage and current at each terminal before and after the fault to locate the fault accurately. Considering the characteristics of the amplitude and phase of the fundamental frequency components, a filtering algorithm taking the average value of multiple measurements to restrain random error is presented as well. A large number of simulation tests verify that the fault location accuracy is not affected by fault type, fault position and fault resistance.

\section{Acknowledgments}

This work was supported by National Key R\&D Program of China (2017YFB0902800) and Science and Technology Project of State Grid Corporation of China (SGTYHT/16-JS-198)

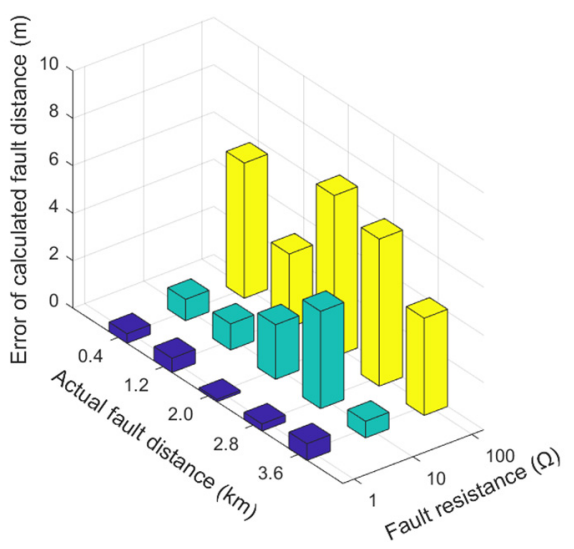

Figure 5. Errors of calculated fault distances for a-b fault

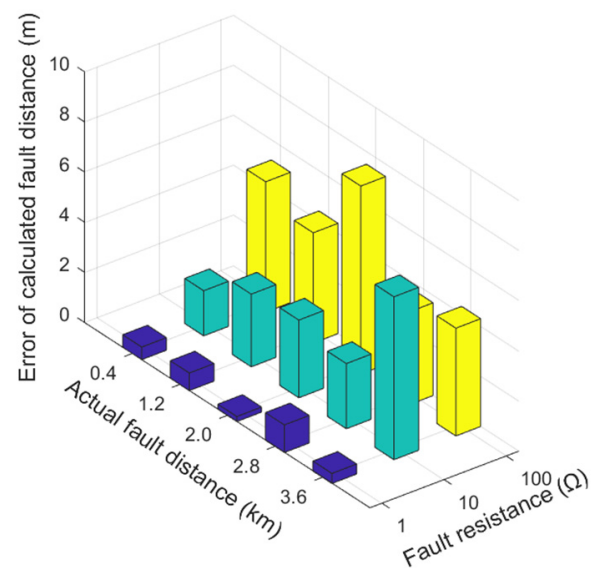

Figure 7. Errors of calculated fault distances for a-b-c fault

\section{References}

1. C. Y. Evrenosoglu, A. Abur. (2005) Travelling wave based fault location for teed circuits. IEEE Trans. Power Del., 20: 1115-1121.

2. Md Shafiullah, Mohammad Ali Abido. (2017) A review on distribution grid fault location techniques, Electric Power Components and Systems, 45: 807824.

3. Y. Liao, S. Elangovan. (2006) Unsynchronized twoterminal transmission line fault location without using line parameters. Proc. Inst. Elect. Eng., Gen. Transm. Distrib., 153:639-643.

4. Y. Liao, N. Kang. (2009) Fault-location algorithms without utilizing line parameters based on the distributed parameter line model. IEEE Trans. Power Del., 24: 579-584.

5. Long Mao, Li Tiangang, An Ranran, et al. (2014) Analysis of line asymmetry parameters' impact on distance protection considering the untransposed transmission line. Power System Protection and Control, 42: 90-94.

6. Dai Zhihui, Chong Zhiqiang, Lichuan, et al. (2015) A New Fault Location Method for Active Distribution Networks Based on Voltage Deviation 
Vector 2- norm. Automation of Electric Power Systems, 39: 96-102.

7. Tao Kai, Liu Mingbo. (2010) Asymmetric issues caused by un-transposed transmission lines and its solution. Power System Protection and Control, 38: 39-43.

8. Ding Hongfa, Duan Xianzhong. (2004) Unbalance issue caused by un-transposed transmission lines and its solution. Power System Technology, 28: 2428,54 .

9. Zhang Qingchao, Yi Lezhao. (2017) Phase-mode transformation of un-transposed transmission lines based on perturbation method. Power System Protection and Control, 45:18-23.

10. Zhang Qingchao, Yi Lezhao, Wang Di, et al. (2018) Application of perturbation method to un-transposed double-circuit transmission lines. Proceedings of the CSU-EPSA, 30:110-116.

11. Lu Jiping, Ye Yilin. (1998) Accurate fault location of transmission lines suitable for any specific structure. Automation of Electric Power Systems, 22: 40-43.

12. Lu Jiping, YE Yilin. (1999) Calculation of Short Circuit faults of transposed and untransposed transmission lines. Journal of Chongqing University, $22: 47-52$

13. Christos A. Apostolopoulos, and George N. Korres. (2010) A Novel Algorithm for Locating Faults on Transposed/Untransposed Transmission Lines Without Utilizing Line Parameters. IEEE Trans. Power Del., 25:2328-2338.

14. Saeed Rezaee1, Saman Babaie-Kafaki1. (2018) A modified nonmonotone trust region line search method, Journal of Computational and Applied Mathematics, J. Appl. Math. Comput.,57:421-436 